\title{
Dynamic Deformation and Failure of Ultrafine-grained Titanium
}

\author{
Zezhou $\mathrm{Li}^{1}$, Bingfeng Wang ${ }^{2}$, Shiteng Zhao ${ }^{1}$, RuslanZ. Valiev ${ }^{3}$,Kenneth S. Vecchio ${ }^{1}$, Marc A. \\ Meyers $^{1 *}$ \\ 1.University of California, San Diego, La Jolla, CA 92093, USA \\ 2.Central South University, Changsha 410083, PR China \\ 3.Institute of Physics of Advanced Materials, Ufa State Aviation Technical University, Ufa 450000, \\ Russia
}

\begin{abstract}
Dynamic deformationand shear localization of ultrafine-grained $(\sim 120 \mathrm{~nm})$ pure titanium areexamined., The strain hardening can be considered as having two regimes: below and above a strain $\sim 0.04$; at this point there is a drastic decrease in the slope.The strain-rate sensitivity of ultrafine-grained titanium was found to be approximately the same as its coarse grained counterpart. Based on experimentally determined parameters, the Zerilli-Armstrong equation is modified to describe the mechanical response of the ultrafine-grained titanium over the strain rate range $10^{-5}$ to $10^{3} \mathrm{~s}^{-1}$. Adiabatic shear banding was examined in a forced shear configuration where large strain is imposed in a narrow region. The microstructure inside the adiabatic shear band consists of a mixture of elongated grains and equiaxed nanograins $(\sim 40 \mathrm{~nm})$ that aresignificantly smaller than the initial grains $(\sim 120 \mathrm{~nm})$. The formation of equiaxed nanograins is modeled through a mechanism of rotational dynamic recrystallization. This further reduction in grain size from the one generated by ECAP is interpreted in terms of the Zener-Hollomon parameters for quasistatic and dynamic deformation. The adiabatic shear band eventually fractures by a combination of brittle and ductile failure.
\end{abstract}

Keywords: Ultrafine-grained titanium; Constitutive response; Adiabatic shear band formation and failure mechanism.

*Corresponding author: Marc A. Meyers; mameyers@eng.ucsd.edu 


\section{Introduction}

Ultrafine-grained (UFG)and nanocrystalline metals have been the subject of widespread research over the past couple of decades with significant acceleration in recent years [1].As the name suggests, nanocrystalline materials are single or multi-phase polycrystals with nano-scale $(1-100 \mathrm{~nm})$ grain size.At the upper limit of this regime, the term "ultrafine grain size (UFG)" is often used (grain sizes of 100-1000 nm) [2].Most studies on the failure behavior of UFG and nanocrystalline materials have mainly focused on the phenomena occurring under static or quasi-static loading, whereas research on dynamic properties under high strain rates is rare.Shear bands are often sites of initiation and growth for subsequent dynamic fracture $[3,4]$.

There have beena number of investigations on the dynamic behaviorof $\alpha$-titanium withdifferent grain sizes.Meyers et al. [5] investigated dynamic response and shear-band formation of CP grade 2 titanium with agrain size $72 \mu$ min high strain-rate plastic deformation. Micrograins smaller than the initial grain size were observed within the shear band and attributed to the dynamic recovery/recrystallization.Koet al.[6] and Liu et al. [7]studied mechanical behavior of UFGpure titanium ( 300nm) and showed that itexhibited a significant improvement in yield strength with an absence of strain hardening behavior compared with coarse grained titanium.However, the dynamicbehavior of UFG titanium with grain size significantly below 300nm, especially shear localization,still remains largely unexamined.

\section{Experimentalprocedure}

The as-received UFGpure titaniumsamples investigated were 200mm long and $6 \mathrm{~mm}$ diameter rods processed by ECAP-C technique, according to a general procedure presented elsewhere [8, 9]. The average grain size of the as-received UFGtitanium was $\sim 120 \mathrm{~nm}$. The microstructure of 
cross-section of as-received titanium rod was observed by Lopeset al. [10] usingTEM as shown in Fig.1. Dynamic compression tests were performed using a split Hopkinson pressure bar (SHPB). The dynamic mechanical properties and shear deformation were measured using cylinder and hat-shaped specimens. The cylinders had a length and diameter of $4 \mathrm{~mm}$. Hat-shaped specimens were used to generate high shear strain in the "forced" localized region [11].Fig.2(a) illustrates their geometry and dimensions. The details and principles of this procedure are discussed by Meyers et al.[11] and have been used for the formation of adiabatic shear bands incoarse grained titanium [12].

The deformedhat-shaped samples were cut parallel to the shear band for microstructural examination. A diamond saw at a speed of $600 \mathrm{rpm}$ and a feed speed of approximately $0.01 \mathrm{~mm} / \mathrm{s}$ was used. The etching solution for UFGtitanium was2.5ml HF, 3ml $\mathrm{HNO}_{3}, 5 \mathrm{ml}$ HCland $91 \mathrm{ml}_{2} \mathrm{O}$.The shear bandwas examined in a Phillips XL30 SEM. A focused ion beam (FIB) instrument was used to accurately prepare TEM samples in the shear band regions. The FIB specimen orientationis shown in Fig.2(b).Figures2(c) and 2(d) further show the FIB sectionsinsamples B and C, which are perpendicular to shear band and along shear band,respectively. The specimens were characterized by TEMusing a FEI TecnaiG ${ }^{2}$ Polara transmission electron microscope operating at300kV.

\section{Results and Discussion}

\subsection{Mechanical properties of UFG titanium}

\subsubsection{Strain hardening and strain-rate sensitivity}

Fig.3(a)showsthe room-temperature compression stress-strain curves at different strain rates. After an initial stage of rapid strain hardening, the strain-hardening rate beyond a strain of 0.04 is significantly reduced.This is characteristic of nanocrystalline and UFG materials, which often show low strain-hardening rate. Fig. 3(b) further shows that the strain-hardening rate of UFG titanium 
under dynamic loading follows the same trend. In contrast, the flow stress of coarse-grained polycrystalline titanium under dynamic loading continues to increase [5]. The density of dislocations in a nanocrystalline metal saturates due to dislocation annihilation at grain boundaries. It thus loses its defect accumulation ability and hence the capacity for strain hardening.However, dislocations are still the carriers of plastic deformation until the grain size is in the range of $10-15 \mathrm{~nm}$.Flat compression curves have been observed for UFG pure titanium made by ECAP [13]. Elias et al.[14] also reported a low strain-hardening rate for UFGtitanium having agrain size 200nmafter a strain of 0.05.

The strain-rate dependence of the yield stress for two grain sizes $(72 \mu$ mand $120 \mathrm{~nm})$ are shown in Fig.3(c). The strain-rate sensitivity, defined as $m=\partial \log \sigma / \partial \log \varepsilon$, is 0.027 between $10^{-4} \mathrm{~s}^{-1}$ and $10^{3}$ $\mathrm{s}^{-1}$ for both UFG titanium and coarse-grained titanium $[5,15]$. At the higher strain rate, there is an increase in $m$. The activation volume parameter concept provides insightinto the deformation mechanisms [16].It is expressed as:

$$
v^{*}=\frac{k T}{m \sigma_{y}}(1)
$$

where $T$ is the temperature, $\sigma_{y}$ is the yieldstress, and $k$ is Boltzmann constant. It can be seen from Eqn. 1 that the activation volume $v^{*}$ is inversely proportional to both the strain-rate sensitivity and yield stress.The yield stress of UFG titanium is approximately twicethat of coarse-grained CP titanium. The activation volume of UFG pure titanium is $40 b^{3}$, where $b=0.289 \mathrm{~nm}$ is the Burgers vector in hexagonal titanium.Thisrepresents a significant reductionintheactivation volume, compared with $80 b^{3}$ for coarse-grained CP titanium [10],suggesting a change in the rate controllingmechanism. The influence of the cutting of forest dislocations decreases for UFG materials.This results in a decrease of the activation volume related to its low strain-hardening response.

\subsubsection{Constitutive response}


Zerilli and Armstrong [17,18] developed physically-based constitutive equationsbased on the general mechanical responses for fcc, bcc and hcp metals.The Zerilli-Armstrong equation is based on the framework of thermally-activated dislocation motion, where both the work hardening and strain-rate sensitivity are influenced by the temperature.This equation was applied byMeyers et al.[19] to describe the mechanical response of hexagonalmaterials and is modified below to account for two regimes:

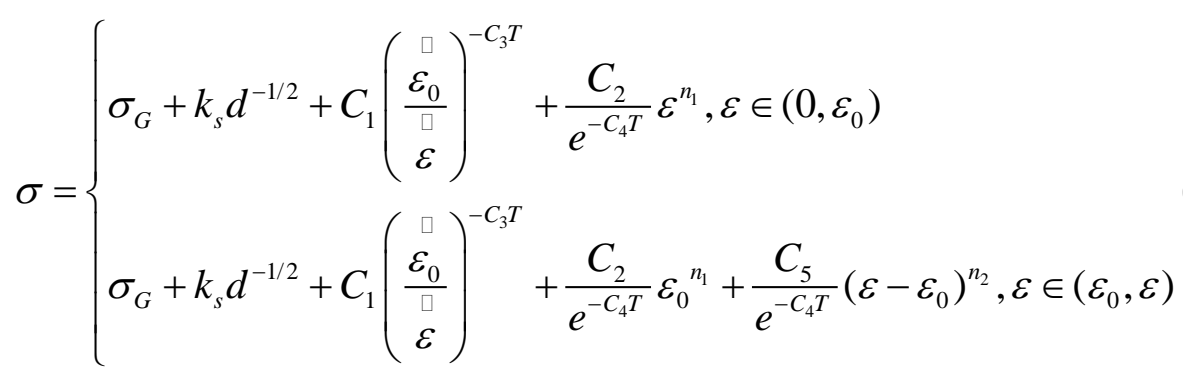

where $\sigma_{G}$ represents the athermal part of the stress required for plastic deformation.The second term in theright-hand side represents the Hall-Petch relationship (also considered athermal). The third and fourth terms represent the increase of theyield stress related to an increase of strain rate $\varepsilon$ and plasticstrain $\varepsilon$, respectively (thermal part). As discussed, in the first stage of plastic deformation, for a strain less than0.04, dislocations are still the carriers of plastic deformation for the UFG titanium.For the nearly zero post-yield work-hardening range( $\varepsilon \geq 0.04)$, the experimentally determined parameters, $C_{5}$ and $n_{2}$, are changed to describe its low strain hardening ability.This provides the fifth term. For UFG titanium, $\sigma_{G}=0 \mathrm{MPa}, k_{s}=6 \mathrm{MPa} \sqcup \mathrm{mm}^{-1 / 2}$ [2], $C_{1}=420 \mathrm{MPa}, C_{2}=2200 \mathrm{MPa}, C_{3}=8.05 \times$ $10^{-3} \mathrm{~K}^{-1}, \quad C_{4}=6.8 \times 10^{-4} \mathrm{~K}^{-1}, C_{5}=655 \mathrm{MPa}, \varepsilon_{0}=1700 \mathrm{~s}^{-1}, \mathrm{n}_{1}=0.33$ and $\mathrm{n}_{2}=0.8$. The validity of theconstitutive equationsis further assessed by comparing the experimental and predicted results in Fig. 3(d).

\subsection{Adiabatic shear band formation and failure}

\subsubsection{Mechanical response and temperature rise in adiabatic shear band}


The thermal softening associated with the plasticity-induced temperature rise may lead to shear localization [20]. The hat-shaped samples H-1,(with maximum displacement of $1 \mathrm{~mm}$ )and H-2 (with maximum displacement of1.15 mm)were loaded at different stresses. The formation of adiabatic shear band in sample $\mathrm{H}-1$ is discussed first;the flow stress versus strain in the ASB for the sample $\mathrm{H}-1$ is shown in Fig.4(a). In the first stage (A-B), the shear stress increases with shear strain due to strain hardening and strain-rate hardening.When the stress reaches the maximum value of 650MPa, itbegins to fluctuate due to the competition between strain hardening, strain-rate hardening and thermal softening;this is the second stage (B-C). In the third stage (C-D), when thermal softening is larger than strain hardening and strain-rate hardening, the stress decreases sharply and an ASB is generated in the specimen.

Assuming adiabatic heating, it is possible to estimate the temperature rise within the shear band.Taking into account that a fraction $\beta$ of the work done is converted into heat, the followingequation for the conservation of energy holds [20]:

$$
\rho \cdot C_{p} \cdot d T=\beta \cdot \sigma \cdot d \varepsilon
$$

where $\rho$ is the density of the material, $C_{P}$ is the specific heat capacity, $T$ is the temperature, $\sigma$ is the true stress and $\varepsilon$ is the strain. The temperature rise is:

$$
\Delta T=\frac{\beta}{\rho \cdot C_{p}} \int_{\varepsilon_{e}}^{\varepsilon_{s}} \sigma d \varepsilon
$$

The initialconditions are $\varepsilon_{s}=0$ and $T=293 \mathrm{~K} ; \beta=0.9, C_{p}=523 \mathrm{~J} /(\mathrm{kg} \cdot \mathrm{K})$, and $\rho=4500 \mathrm{~kg} / \mathrm{m}^{3}$. The temperature increase as a function of the shear strain in the shear band for UFG titanium is shown in Fig. 4(a). The recrystallization temperature $T=900 \mathrm{~K} \approx 0.46 T_{m}$ can be reached at a shear strain $\sim 4.5$ (This is obtained by converting the normal strain into shear strain).

\subsubsection{Width and susceptibility of formation of shear band}


A theoretical shear-band thicknessequation wasobtained by Bai and Dodd [21]:

$$
\delta=2\left(\frac{\lambda T}{\tau \gamma}\right)^{1 / 2}
$$

where $\lambda=20 \mathrm{~W}(\mathrm{~m} \cdot \mathrm{K})^{-1}, T=900 \mathrm{~K}$, and $\gamma=5 \times 10^{4} \mathrm{~s}^{-1} . \lambda, \mathrm{T}, \quad \gamma$ and $\tau$ are the thermal conductivity, temperature, shear strain rate and shear stress inside the shear band, respectively.Grady[22, 23] proposed an alternative equation:

$$
\delta=\left(\frac{16 \rho^{3} C_{p} \chi^{3}}{\tau^{3} \alpha^{2} \gamma}\right)^{1 / 4}
$$

where $\alpha$ is the thermal softening parameter, and $\chi$ is thermal diffusivity coefficient.ForUFGtitanium, $\rho=4500 \quad \mathrm{~kg} / \mathrm{m}^{3}, \quad \chi=\lambda /\left(\rho \cdot C_{p}\right)=8.5 \times 10^{-6} \quad \mathrm{~m}^{2} / \mathrm{s}, \quad C_{p}=523 \quad \mathrm{~J} /(\mathrm{kg} \cdot \mathrm{K}), \quad \gamma=5 \times 10^{4} \mathrm{~s}^{-1} \quad$ and $\alpha=-\left(1 / \sigma_{y}\right)[\partial \sigma / \partial T=0.002$ [10].Fig.4(b)shows predictions from the above equations and the experimentallymeasured shear band thickness of UFGand coarse-grained titanium; the shear-band thickness measured from Fig.2(b) is only 2 $\mu$ m.Mishra et al. [24] reported that there was a large drop of shear-band thickness forUFG copper compared with its coarse grained counterpart. The reason for this difference is considered to be the increased propensity to thermal softening in ECAP samples.Although both the Bai-Dodd and Grady equations predict a decrease in band thickness with increasing flow stress, the precipitous drop for the UFG titanium must be attributed to an additional mechanism for the loss of mechanical stability.Guo et al.[25] reported that the thermal conductivity of nanocrystalline titanium with a grain size less than $100 \mathrm{~nm}$ is $5.2(\mathrm{~m} \cdot \mathrm{K})^{-1}$, which is only about one fourththat of the coarse-grained titanium $20(\mathrm{~m} \cdot \mathrm{K})^{-1}$. The thermal softening parameter of UFG titanium is twice that of coarse-grained titanium [5,10]. From Equations5 and 6, the decrease of thermal conductivity parameter $\lambda$ and increased thermal softening parameter $\alpha$ explain the decrease 
in shear-band thicknesscompared with coarse grained titanium.

A quantitative description of the susceptibility to adiabatic shear banding (ASB), $\chi_{S B}$, by Wright [26,27], has the followingform:

$$
\frac{\chi_{S B}}{a / m}=\min \left\{1, \frac{1}{n / m+\sqrt{n / m}}\right\}(7)
$$

where $a$ is a nondimensional thermal softening parameter defined by $a=(-\partial \sigma / \partial T) / \rho C_{p}, n$ is the strain hardening exponent and mis the strain-rate sensitivity.For a perfectly plastic material (no strain hardening), the susceptibility reduces to:

$$
\chi_{S B}=\frac{a}{m}=\frac{\alpha \sigma_{y}}{\rho C_{p} m}(8)
$$

where $\alpha=0.002 \mathrm{~K}^{-1}$ is the thermal softening parameter evaluated under isothermal conditions and $\sigma_{y}$ is the yield strength [5]. The susceptibility to ASB of the UFG titanium can thus be estimated to be $\sim 30$ using the following parameters: $\sigma_{y}=0.97 \mathrm{GPa}, m=0.027$ [10].For the coarse grained titanium, $\sigma_{y}$ $=0.4 \mathrm{GPa}, \alpha=0.001 \mathrm{~K}^{-1}, m=0.027$ [5]results in a susceptibility to ASB of $\sim 6$, five times lower than that for the UFG Ti. A similar increase in susceptibility to shear localizationof UFG Fewas also reported by Wei et al. [28].

\subsubsection{Examination of the microstructure}

TEM was carried out on the samples B and C to study microstructural features of the shear bands. Fig.5(a) providesan overall view of sample B (the shear band lies between the blue lines). The microstructures within and outside the shear band are quite distinct.The continuous sharp diffraction ring pattern indicates that the microstructure is randomly oriented,with no strong texture. Lins et al. [29] showed thatthe shear band in an interstitial-free steel displays a strong heterogeneouscharacter from the center of the shear band to its edges.Fig.5(b) shows both the nominally equiaxed UFGmatrix 
and abnormallygrown coarse grainswith low dislocation density (see arrow). The abnormal grain growth is due to the temperature rise near and in the shear band and the thermal instability of the UFG titanium. The elongated grains, which wereless severely deformed, were formed along the edge of the shear band. Fig.5(c) shows a mixture of elongated and equiaxed grains inside the shear band. The grain boundaries are clearly delineated. The thickness of the elongated grains varies, which may be due to the inhomogeneous shear stressdistribution inside the shear band. Some very narrow elongated grains (see arrow)are formed, which may further break down to form nanograins upon further deformation. This evolution process was described inseveral studies[5,11].Fig.6(a)shows themicrostructure of sample C. It shows the randomly distributed nanostructured grains. The dark field imageof Fig.6(b) shows that the nanograins tend to flow in one direction (shown by arrows) under shear deformation inside the shear band. Fig.6(c)highlights a high magnification image of a nanograinwith grain size $\sim 40 \mathrm{~nm}$, corresponding to the breakdown of the narrow elongated grains as detailed in Fig. 6(c).

In Fig. 5, the FIB cutting direction is perpendicular to the shear band; therefore, the sample is only partially in the shear band (shown between two blue lines). The matrix of UFG titanium can also be seen. Both elongated UFG grains and recrystallized nanosized grains can be observed. Fig. 6, on the other hand, only shows the microstructure right in the middle of the shear band, since the FIB cutting direction is parallel to the shear band. Only fully recrystallized nanograins are seen. The temperature rise in the middle of the shear band is higher than that at its edges, providing more thermal energy to form recrystallized equiaxed nanograins. Different applied shear strains and inhomogeneous temperature distribution occur during the formation of the shear band, as demonstrated by finite element simulation of formation and failure of the shear band [30]. This leads 
to different shear direction and different microstructures in different regions as shown in Figs. 5 and 6. As indicated by the TEM diffraction ring pattern in Figs. 5 and 6, the recrystallized grains are nearly randomly distributed, and do not show strong texture inside the shear band.

\subsubsection{Modeling of microstructural evolution inside the shear band}

Meyers et al. [31], and Hines and Vecchio [32] demonstrated that migrational recrystallization could not account for the grains generated inside the shear band and proposed complementary rotational recrystallization mechanisms. Whereas Meyers et al. [31, 33] used a dislocation energetics approach, Hines and Vecchio [32] and Hineset al. [34] implemented a continuum mechanics analysis. The sequence of events shown in Fig.7 leading to the formation of equiaxed nanograins is expected to occur. The formation of elongated grains is due to the dislocation motion instead of dynamic recrystallization.In essence, the equiaxed grains shown in Fig. 7(a) are deformed into elongated grains as shown in Fig. 7(b). Fig. 7(c) shows that dislocations are accumulated in subgrain boundaries inside the elongated grains. The elongated grains then break up to finer subgrains. Once the elongated subgrains are achieved, further break-up by dislocation generated at the grain boundaries is due to geometrically-necessary dislocations required to accommodate further plastic deformation. The relaxation of elongated subgrains into an equiaxed crystalline structure can occur by minor rotations of the grain boundaries in Fig. 7(d).

According to the rotational dynamic recrystallization mechanism, the formation of a new grain requires local grain-boundary segments to tilt about $30^{\circ}$ during the last stage of the deformation process (from Fig. 7c to 7d)[33]. The time needed for these processes is given by:

$$
t=\frac{\operatorname{LkTf}(\theta)}{4 \delta \eta D_{b 0} \exp \left(-Q_{b} / R T\right)}(9)
$$

where $t$ is time, $L$ is the average subgrain diameter, $\delta$ is grain-boundary thickness, $\eta$ is the grain 
boundary energy, $D_{b 0}$ is a constant related to grain boundary diffusion, $Q_{b}$ denotes the activation energy for grain boundary diffusion, $\theta$ is the subgrain misorientation, and $f(\theta)$ can be described as follows:

$$
f(\theta)=\frac{3 \tan (\theta)-2 \cos (\theta)}{3-6 \sin (\theta)}+\frac{2}{3}-\frac{4 \sqrt{3}}{9} \ln \frac{2+\sqrt{3}}{2-\sqrt{3}}+\frac{4 \sqrt{3}}{9} \ln \frac{\tan (\theta)-2-\sqrt{3}}{\tan (\theta)-2+\sqrt{3}}
$$

For UFG titanium, $\delta=1.5 \times 10^{-9} \mathrm{~m}, \eta=0.675 \mathrm{~J} / \mathrm{m}^{2}, D_{b 0}=1.4 \times 10^{-3} \mathrm{~m}^{2} / \mathrm{s}, Q_{b}=151 \mathrm{~kJ} / \mathrm{mol}, k=$ $1.38 \times 10^{-23} \mathrm{~J} / \mathrm{K}, R=8.314 \mathrm{~J} /(\mathrm{K} \cdot \mathrm{mol}), T=900 \mathrm{~K}[35,36,37]$. The kinetic curve for the rotational dynamic recrystallization mechanism in a shear band can be obtained by incorporating the parameters into Eqns. 9 and 10, as shown in Figs.8(a) and 8(b).A decrease in theactivation energy for grain-boundary diffusion[10] and increase of grain-boundary thickness [35] accelerate the formation of equiaxed grains by the grain-boundary diffusion process. In Fig. 8(a), the nanograin size, $L$, is varied from $40 \mathrm{~nm}$ to $100 \mathrm{~nm}$ at $T=900 \mathrm{~K} \approx 0.46 T_{m}$. Fig. $8(\mathrm{~b})$ indicates that the increase of temperature helps the grain-boundary diffusion process.The rate of rotation decreases with increasing angle and asymptotically approaches $30^{\circ}$ as $t \rightarrow \infty$.The calculationspredict significant rotations of the boundarywithin the deformation time.Thus, rotational recrystallization can also take place duringplastic deformation $\left(2 \times 10^{-4} \mathrm{~s}\right)$. This does not exclude the possibility of further coalescence of nanograins inside the shear band. Since the deformation mechanisms innanocrystalline titanium are different from the ones in coarse-grained titanium, the discussion below provides deeper insight into the formation of nanograins inside the shear band.

\subsubsection{Grain boundary mobility}

The driving forcefor the migration of a grain boundary can be simply estimated by equatingthe force acting on a curved segment of the boundary, $d S$, due to thegrain-boundary energy $\eta=0.675 \mathrm{~J} / \mathrm{m}^{2}$ [37].Assuming a two-dimensional geometry, for simplicity, the total force acting on thegrain boundary in the y-direction (seen inFig.8(c)) is: 


$$
F=2 \eta \sin \frac{d \theta}{2} \approx \eta d \theta
$$

Therefore,

$$
\text { force / area }=\frac{\eta d \theta}{R d \theta} \approx \eta d \theta(12)
$$

The velocity of the grain boundary is proportional to theforce acting on the segment $d S$ :

$$
v \propto \frac{\eta}{R}=M \frac{\eta}{R}(13)
$$

where $M$, the grain boundary mobility,has an Arrhenius-type temperature dependence [38]:

$$
M=M_{0} \exp \left(\frac{-Q_{m}}{R T}\right)
$$

For titanium [39], $M_{0}=4.3 \times 10^{-6} \mathrm{~m}^{4} / \mathrm{J} / \mathrm{s}$ and $Q_{m}=179 \mathrm{KJ} / \mathrm{mole}$.

Grain-boundary velocity is plotted against the grain size at 900K in Fig.8(d).For agrain size of $40 \mathrm{~nm}(v=6 \mathrm{~nm} / \mathrm{s})$, a grain boundary movement of $6 \mathrm{~nm}$ at $900 \mathrm{~K}$ can be expected in $1 \mathrm{~s}$. This cannot be sufficient to reorganize the deformed grain configuration.For a shear band, the cooling takes place in time scales of fractions of milliseconds. For $0.2 \mathrm{~ms}$ and a grain size of $40 \mathrm{~nm}$, a displacement of $1.2 \times$ $10^{-3} \mathrm{~nm}$ is obtained. Thus, the calculation shows that no significant grain growth by grain-boundary migration takes place after a nanocrystalline size of $40 \mathrm{~nm}$ is reached. However, growthof equiaxed nanograins might occur by grain rotation during shear deformation inside the band.

\subsubsection{Zener-Hollomon parameter and grain refinement}

Mishra et al. [24] reported that the similarity of structure evolution in SPD and shear-band formation is related to the hypothesis that both processes are regulated by a similar mechanism with two significant differences: the time is shorter and temperature is higher in shear-band formation. The Zener-Hollomon (ZH) parameter is defined as [40]:

$$
Z=\varepsilon \exp \left(\frac{-Q_{d}}{R T}\right)
$$


where $R$ is the gas constant and $Q_{d}$ is the related activation energy for deformation.At a constant $Z$, the resultant grain size in SPD and dynamic deformation is the same; this is clear from the opposing effects of $\dot{\varepsilon}$ and $T$ in Eqn. 15.Li et al. [41] reported that finer copper grains were formed in deformation at a temperature of77K at high strain rates, on the order of $10^{2} \mathrm{~s}^{-1}$, than under quasi-static conditions. This increased the ZH parameter of the process and consequently decreased the resulting grain size. Extending the proposal of Mishra et al. [24], they reported thatthe recrystallized grain size was inversely related to the ZHparameter.

In fact, a different grain refinement mechanism inside the shear band for nanocrystallinetitanium mayoperate at the high Zener-Hollomon parameterdeformation conditions.For nanocrystalline titanium, as grain size decreases,an increasing amount of grain-boundary sliding can occur. Using MD simulation,Schiøtzet al. [42] reported that grain-boundary sliding dominates the plastic deformation instead of dislocation motion, due to the larger volume fraction of grain-boundaries when the grain size is under $10 \mathrm{~nm}$. Thus, withfurther increase of the $\mathrm{ZH}$ parameter, the grain size inside the shear band may reach a steady-state value of several nanometers, where most plastic deformation is due to grain-boundary sliding instead of dislocation motion.

\subsubsection{Fracture of adiabatic shear band}

The formation of an ASB precedes the failure mechanisms by void and crack formation.Xue et al.[43] observed both elongated and equiaxed voids that preceded failure of the shear band. Both morphologies are observed inside the shear bands, depending on how the separation of the two parts occurs. If only tension is applied, the two parts separate by forming voids in the shear band; thesevoids grow until their diameters reach the band thickness. The elongated dimples are a characteristic of shear failure, due to elongated and coalesced voids. The formation of elongated voids 
when the failure has a strong shear component is explainedby Meyers and Chawla [44].

Fig. 9(a) shows a microvoidin sample H-1 between the shear band and its surrounding; this indicates that microvoids can be nucleated at the edge of the shear band.Fig. 9(b) shows the ductile ligaments (see arrow) along the shear band in sample $\mathrm{H}-1$. The ductile ligaments indicate the coalescence of microvoids. The shear failure surface of the sampleH-2 (which underwent a higher strain than H-1) was observed by SEM shown in Fig. 9(c). The fracture surface contains both ductile features such as dimples,and brittle features such as cleavage patterns,indicating that brittle and ductile fracture modes might take place simultaneously. A microstructure consisting of elongated dimples, shows similar characteristics to other HCP materials, such as Re, Hf and Ti-6Al-4V alloy [45]. The coalescence of voids, leading to cracks, was observed along the shear band in rhenium in high-strain-rate deformation [46]. Similar elongated shear dimples were observed in the fracture surface of polycrystalline hafnium [47].

A schematic illustration of ASB fracture is shown in Fig.9(d).Microvoids can be nucleated inside shear band or at its edges, followed bytheir coalescenceand the formation of microcracks;this leads to the formation of a mixture of ductile and brittle failure. The formation of cracks between sheets of dimples along the shear band can lead to more rapid fracture of the ASBand amore brittle fracture.

\section{Conclusions}

The present investigation addressed the dynamic deformation, adiabatic shear-band formation, and failure mechanism of ultrafine-grained (grain size 120nm) titanium. The conclusions are as follows:

(1) The flow stress of UFG titanium at different strain ratesis described by a modified Zerilli-Armstrong constitutive equation. After an initial stage state of rapid stress rise, the strain 
hardening beyond a strain of 0.04 is considerably lower.The strain-rate sensitivity of UFG titaniumis similar to the one in coarse grained CP titanium.

(2) The width of the shear bandformed by forced shear stress in UFG titanium is about $2 \mu \mathrm{m}$, which is

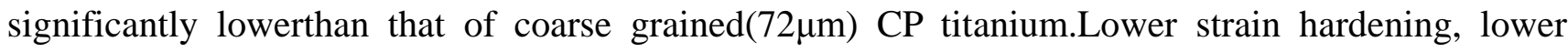
thermal conductivity and higher thermal softening make UFG titanium more sensitive to shear localization and lead to the decrease in the width of the shear band.

(3) Randomly orientednanograins were observed inside the shear band. The equiaxed nanograins, which are under 100nm,are generated inside the shear bandby a mechanism of rotational dynamic recrystallization.

(4) The basic equations of rotational dynamic recrystallization are applied to the nanograins and it is demonstrated that the grain boundaries can undergo $30^{\circ}$ rotation during deformation, thus enabling a steady-state process, in which the equiaxed structure is retained.

(5) The adiabatic shear band eventually fractures by a combination of brittle and ductile failure.The microvoids can be nucleated both inside the shear bandsand attheir edges,followed bytheir coalescenceand crack formation linking them. The formation of cracks along the shear band can lead to more rapid failure of the ASB anda more brittle fracture.

\section{Acknowledgements}

We thank the Department of Energy NNSA/SSAP (DE-NA0002080) for partial support and a UC Research Laboratories Grant (09- LR-06-118456-MEYM). Support ofZezhou Li by China Scholarship Council (201508020004) is greatly acknowledged.The help provided byDr. T. Remington in preparingthe TEM samples by FIB and of H.C. Quan inSEM is greatly appreciated.

\section{References}


[1] C.Suryanarayana, Nanocrystalline materials,Int.Mater. Rev. 2 (1995)40-41.

[2] M.A. Meyers, A. Mishra, D.J. Benson, Mechanical properties of nanocrystalline materials, Prog. Mater. Sci.51 (2006)427-556.

[3] Y.G. Kim, B. Hwang, S. Lee, D.H. Shin, Dynamic deformation and fracture behavior of ultra-fine-grained pure copper fabricated by equal channel angular pressing, Mater. Sci. Eng. A 504 (2009) 163.

[4] A.J. Rosakis, G. Ravichandran, Dynamic failure mechanics, Int. J. of Solids and Structures,37 (2000) 331-332.

[5]M.A. Meyers, G. Subhash, B.K. Kad, L. Prasad, Evolution of microstructure and shear-band formation in $\alpha$-hcp titanium,Mech. of Mater.17 (1994) 175-193.

[6] Y.G. Ko, D.H. Shin, K.T. Park, C.S. Lee, An analysis of the strain hardening behavior of ultra-fine grain pure titanium, Scr. Mater.54 (2006) 1785-1789.

[7] J. Liu,A.S. Khan, L. Takacs, C.S. Meredith, Mechanical behavior of ultrafine-grained/nanocrystalline titanium synthesized by mechanical milling plus consolidation: Experiments, modeling and simulation,Int. J.of Plasticity64 (2015) 151-163.

[8]C. Xu, S. Schroeder, P.B. Berbon, T.G.Langdon,Principles of ECAP-Conform as a continuous process for achieving grain refinement: Application to an aluminum alloy, Acta Mater58 (2010) 1379-1386.

[9] R.Z. Valiev, T.G.Langdon, Achieving exceptional grain refinement through severe plastic deformation: New Approaches for Improving the Processing Technology,Metall. Mater. Trans. A42 (2011) 2942-2952.

[10] F.P.D. Lopes, C.H. Lu, S. Zhao, S.N. Monteiro, M.A. Meyers,Room temperature dynamic strain 
aging in ultrafine-grained titanium, Metall. Mater. Trans. A46 (2015) 4468-4477.

[11] M.A. Meyers, Y. Xu, Q. Xue, M. Perez-Prado, T. McNelley, Microstructural evolution in adiabatic shear localization in stainless steel, Acta Mater.51 (2003) 1307-1325.

[12] M.A. Meyers, H.-R. Park, Observation of an adiabatic shear band in titanium by high-voltage transmission electron microscopy,Acta Mater.34 (1986) 2493-2499.

[13] D. Jia, Y.M. Wang, K.T. Ramesh, E. Ma, Y.T. Zhu, R.Z. Valiev, Deformation behavior and plastic instabilities of ultrafine-grained titanium, Appl. Phys. Lett. 79 (2001) 611-613.

[14] C.N. Elias, M.A. Meyers, R.Z. Valiev, S.N. Monteiro, Ultrafine grained titanium for biomedical applications: An overview of performance, J. Mater. Res. Tech. 2 (2013) 340-350.

[15] I. Weiss, S.L. Semiatin,Thermomechanical processing of alpha titanium alloys—an overview, Mater. Sci. Eng. A263 (1999) 243-256.

[16] L. L, R. Schwaiger, Z.W. Shan, M. Dao, K. Lu, S. Suresh. Nano-sized twins induce high rate sensitivity of flow stress in pure copper, Acta Mater.53 (2005) 2169-2179.

[17] F.J. Zerilli, R.W.Armstrong,Dislocationmechanicsbased constitutive relations for material dynamics calculations, J. Appl. Phys.61 (1987) 1816.

[18] F.J. Zerilli, R.W. Armstrong.Description of tantalum deformation behavior by dislocation mechanics based constitutive relations,J. Appl. Phys.68 (1990) 1580.

[19] M.A. Meyers, O. Vöhringer and V.A. Lubarda.The onset of twinning in metals: a constitutive description, Acta Mater.49 (2001) 4025-4039.

[20] M.A. Meyers, Dynamic Behavior of Materials, Wiley, New York, 1994.

[21] B. Dodd, Y.L. Bai,Width of adiabatic shear bands found under combined stress, Mater. Sci. Technol. 5 (1989) 557-559. 
[22] D.E. Grady,Dissipation in adiabatic shear bands, Mech. of Mater.17 (1994) 289.

[23]D.E. Grady, in“TMS symposium on dynamic behavior of materials”, Warrendale, PA, TMS; 2007.

[24] A. Mishra, M. Martin, N.N. Thadhani, B.K. Kad, E.A. Kenik, M.A. Meyers, High-strain-rate response of ultra-fine-grained copper,Acta Mater.56 (2008) 2770-2783.

[25] F.A. Guo, K.Y. Zhu, N. Trannoy,J. Lu, Examination of thermal properties by scanning thermal microscopy in ultrafine-grained pure titanium surface layer produced by surface mechanical attrition treatment, Thermo. Acta419 (2004) 239-246.

[26] T.W.Wright, The physics and mathematics of adiabatic shear bands, Cambridge: Cambridge Press; 2002.

[27] T.W. Wright, Shear band susceptibility: work hardening materials, Int. J. of Plasticity 8 (1992) 583.

[28] Q. Wei, L. Kecskes, T. Jiao, K.T. Hartwig, K.T. Ramesh, E. Ma, Adiabatic shear banding in ultrafine-grained Fe processed by severe plastic deformation,Acta Mater.52 (2004) 1859-1869.

[29] J.F.C. Lins, H.R.Z. Sandim, H.-J. Kestenbach, D. Raabe, K.S. Vecchio, A microstructural investigation of adiabatic shearbands in an interstitial free steel, Mater. Sci. Eng. A 457 (2007) 205-218.

[30] X. Teng, T. Wierzbicki, H.Couque,On the transition from adiabatic shear banding to fracture, Mechanics of Materials 39 (2007) 107-125.

[31] M.A. Meyers, J.C. LaSalvia, V.F. Nesterenko, Y.J. Chen, B.K. Kad, in: T.R. McNelley (Ed.), Dynamic recrystallization in high strain rate deformation, Recrystallization and Related Phenomena, Rex’96, Monterey, (1997) 279-286. 
[32]J.A. Hines and K.S. Vecchio, Recrystallization kinetics within adiabatic shear bands, Acta Metall. 45 (1997) 635-649.

[33] M.A. Meyers, V.F. Nesterenko, J.C. LaSalvia, Q. Xue, Shear localization in dynamic deformation of materials: microstructural evolution and self-organization, Mater. Sci. Eng. A 317 (2001) 204-225.

[34] J.A. Hines, K.S. Vecchio and S. Ahzi, A model for microstructure evolution in adiabatic shear bands, Mater. Trans. A 29 (1998) 191-203.

[35] X. Sauvagea,G. Wilde, S.V. Divinski, Z. Horita, R.Z. Valiev,Grain boundaries in ultrafine grained materials processed by severe plastic deformation and related phenomena, Mater.Sci.Eng. A 540 (2012) 1-12.

[36] R.A. Perez, H. Nakajima, F. Dyment. Diffusion in $\alpha$-Ti and Zr, Mater.Trans.44 (2003) 2-13.

[37] T.A. Roth, P. Suppayak. The surface and grain boundary free energies of pure titanium and the titanium alloy Ti-6Al-4V, Mater.Sci. Eng.35 (1978) 187-196.

[38] F.J. Humphreys, M. Hatherly,Recrystallization and related annealing phenomena, Oxford: Pergamon Press 1995.

[39] M. Hoseinia, M.H. Pourian, F. Bridier, H. Vali,J.A. Szpunar, P. Bocher,Thermal stability and annealing behaviour of ultrafine grained commercially pure titanium, Mater. Sci.Eng. A 532 (2012) 58-63.

[40] C. Zener, J.H. Hollomon, Effect of strain rate upon plastic flow of steel, J. Appl. Phys. 15 (1944) 22.

[41] Y.S. Li, Y. Zhang, N.R. Tao, K. Lu,Effect of the Zener-Hollomon parameter on the microstructures and mechanical properties of $\mathrm{Cu}$ subjected to plastic deformation, Acta Mater.57 
(2009) 761-772.

[42] J. Schiøtz, K.W. Jacobsen, A maximum in the strength of nanocrystalline copper, Science301 (2003) 1357-1359.

[43] Q.Xue, M.A. Meyers, V.F. Nesterenko, Self-organization of shear bands in titanium and Ti-6Al-4V alloy, Acta Mater.50 (2002) 582-583.

[44] M. A. Meyers and K. K. Chawla, Mechanical Behavior of Materials, Second Ed., Cambridge U. Press, (2009) 476-477.

[45] Y. Bai, Q. Xue, Y. Xu, L. Shen, Characteristics and microstructure in the evolution of shear localization in Ti-6A1-4V alloy, Mech. of Mater. 17, (1994) 155-164.

[46] B. J. Koeppel, G. Subhash, Influence of cold rolling and strain rate on plastic response of powder metallurgy and chemical vapor deposition rhenium, Metall. Mater. Trans. 30 (1999) 2641-2648.

[47] G. Subhash, J. Pletka, G. Ravichandran, Plastic deformation of hafnium under uniaxial compression, J. of Mater. 28 (1997) 1479-1487. 


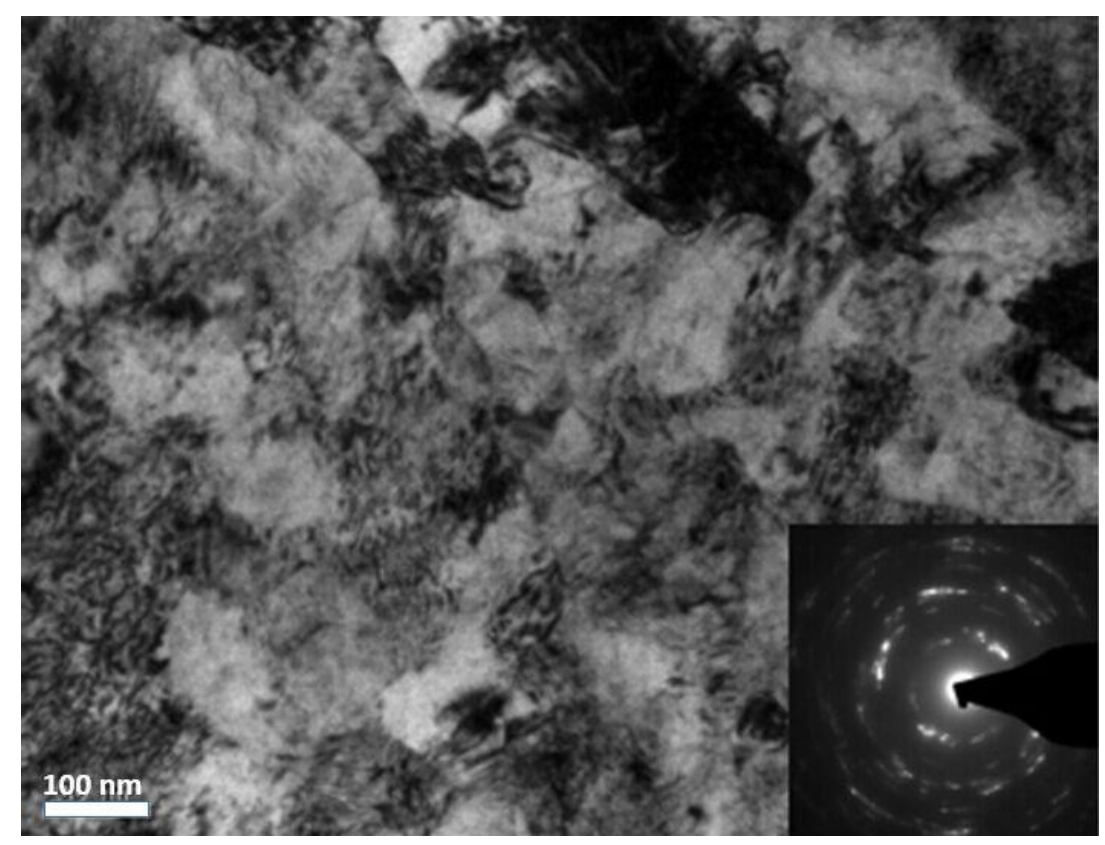

Figure 1. TEM image of the as-received titanium rod [10].
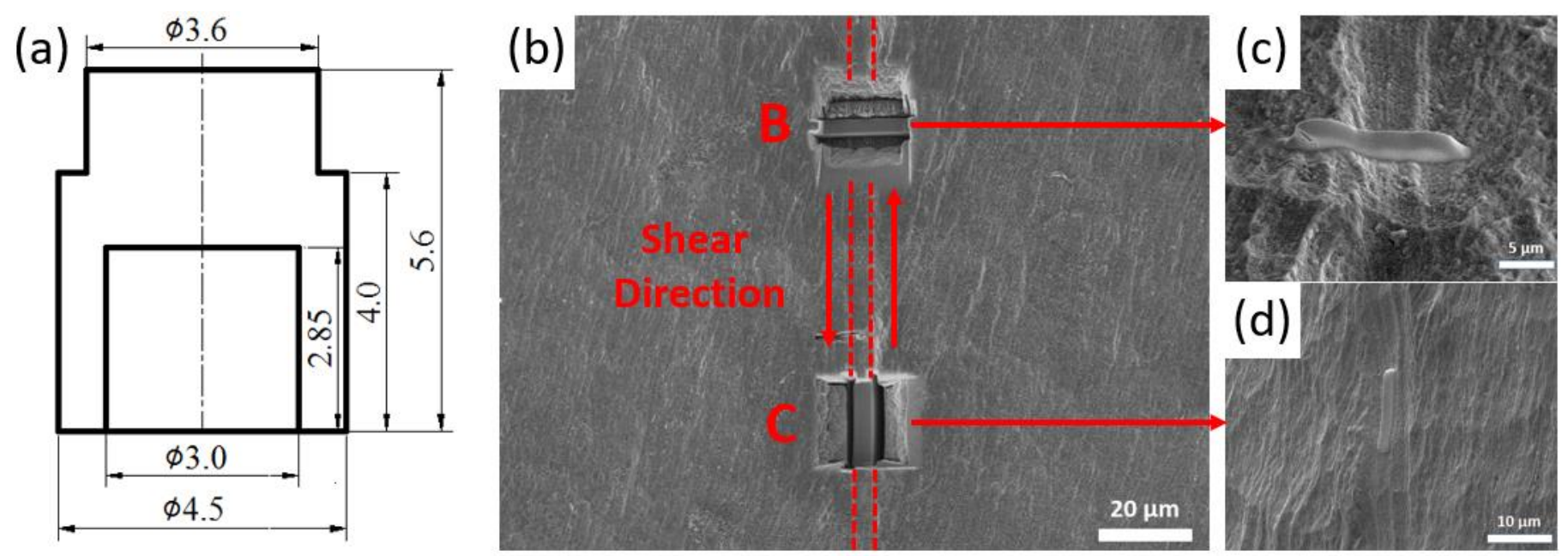

Figure 2. (a) Schematic configuration of the hat-shaped specimen (Dimensions in mm). (b) SEM images of FIB positions. (c) Sample B position perpendicular to the shear band. (d) Sample C position along the shear band. (The edges of the shear band marked by red lines). 

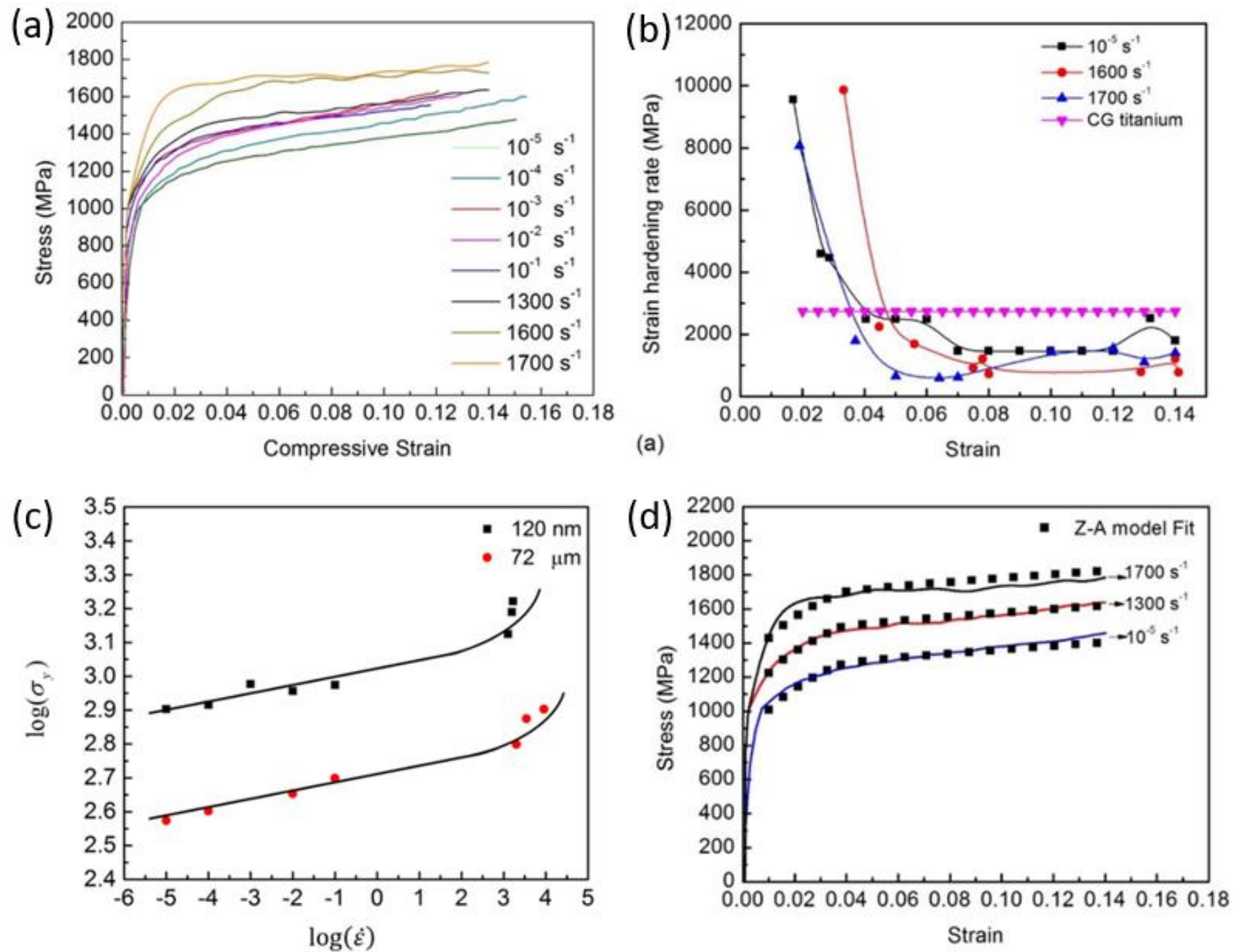

Figure 3. (a) Stress-strain relationships of UFG titanium cylindrical samples at different strain rates [10]. (b) Strain hardening rate as a function of strain [5]. (c) Strain rate sensitivity of coarse grained titanium [5] and UFG titanium [10]. (d) Stress-stress relationship and Zerilli-Armstrong constitutive equation fit to experimental results at strain rates $10^{-5} \mathrm{~s}^{-1}, 1300 \mathrm{~s}^{-1}$ and $1700 \mathrm{~s}^{-1}$. 

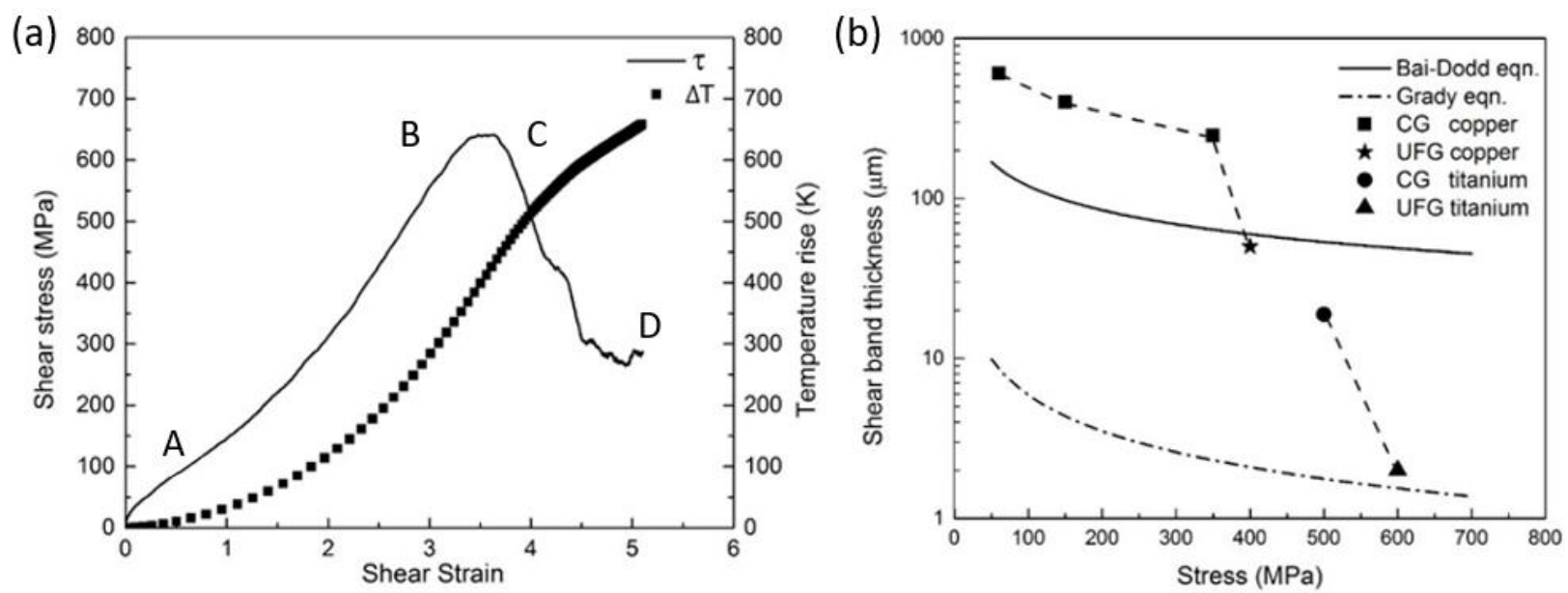

Figure 4. (a) Shear stress versus shear strain in hat-shaped sample with maximum displacement of $1 \mathrm{~mm}$ deformation and corresponding temperature rise. Shear stress-shear strain has three stages: (A-B) flow stress increases with strain due to strain hardening and strain-rate hardening, (B-C) strain hardening, strain-rate hardening and thermal softening compete, (C-D) thermal softening dominates hardening and the shear band is formed. (b) Shear band thickness of copper and titanium with different grain sizes obtained from experiments [5,24] as compared with predicted equations by Bai and Dodd [21] and Grady [22,23]. 


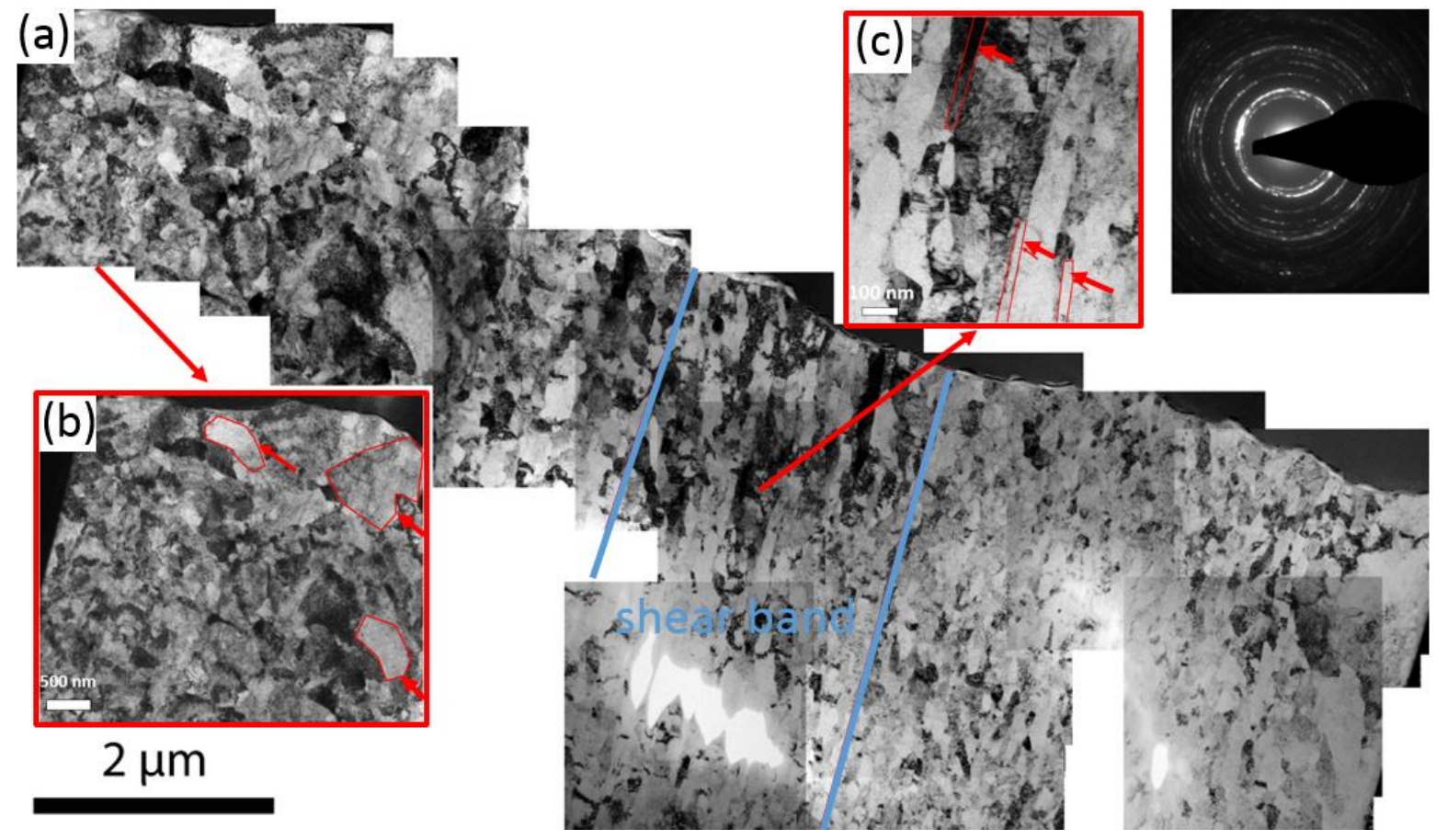

Figure 5. TEM images of the microstructure (a) of the sample B (perpendicular to shear band), (b) near the shear band, (c) inside the shear band. (Shear direction nominally perpendicular to the foil; misorientation is possible). 

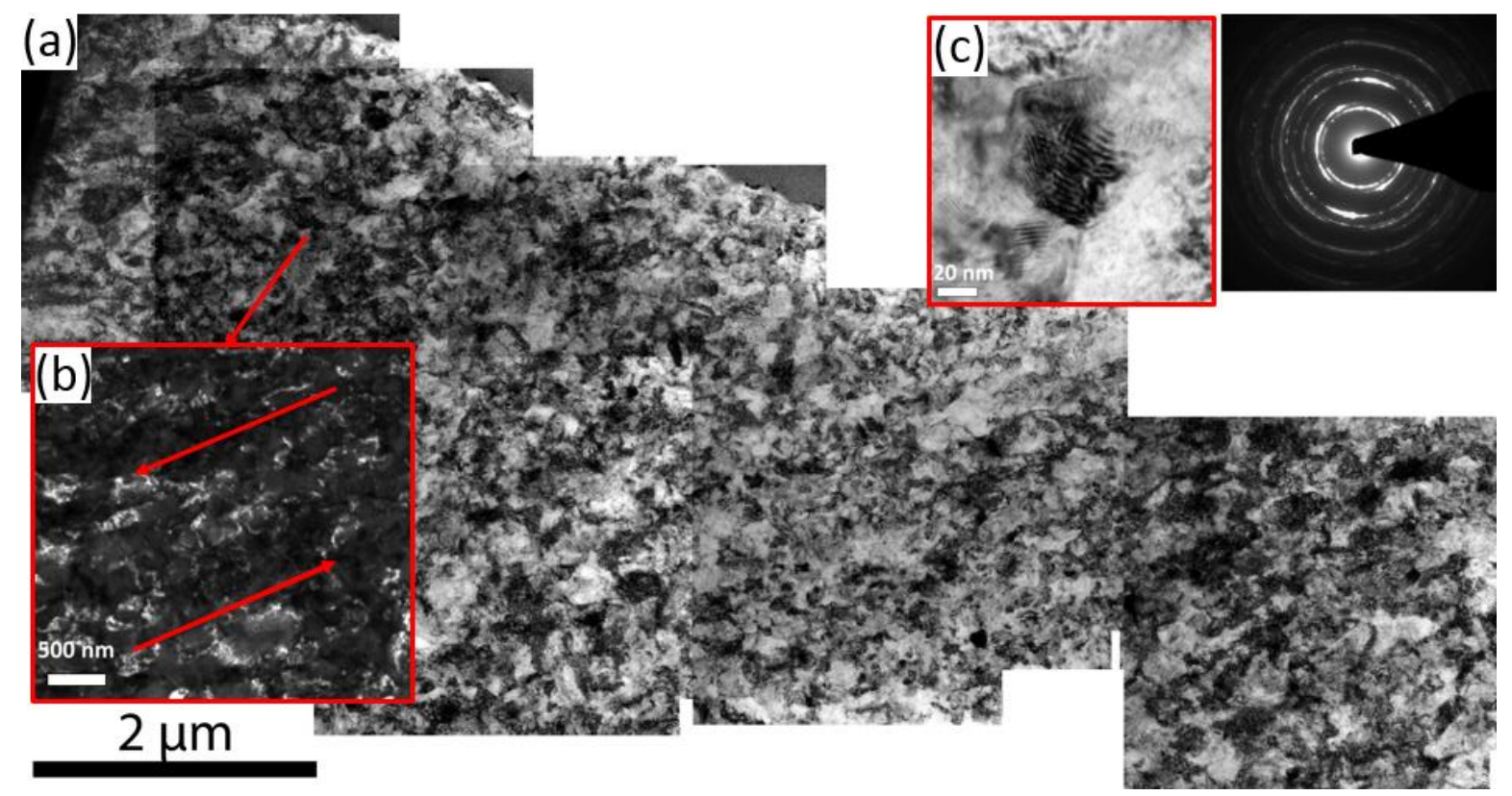

Figure 6. TEM images of (a) the microstructure of the sample C (inside shear band), (b) the dark-field microstructure, (c) the equiaxed grains. (Shear direction indicated in dark-field micrograph (b)). 


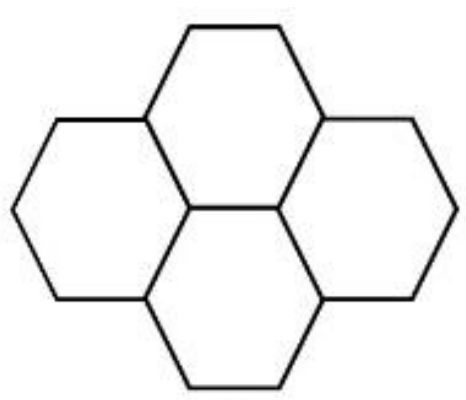

(a)

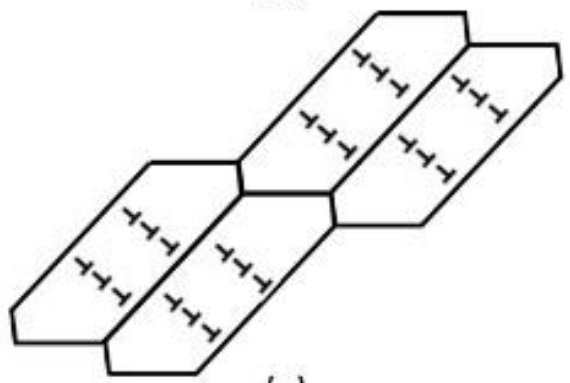

(c)

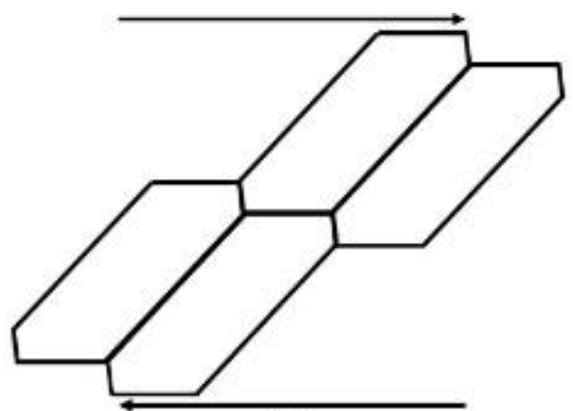

(b)

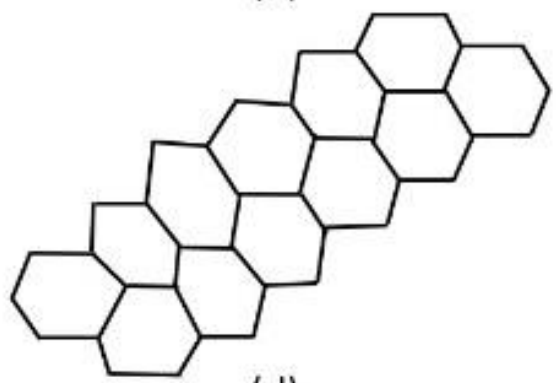

(d)

Figure 7. Schematic illustration of microstructural evolution inside the shear band: (a) randomly distributed equiaxed grains; (b) formation of elongated grains through plastic deformation (shear direction indicated by arrows); (c) dislocations accumulation forming subgrain boundaries; (d) breakup of elongated grains, reorientation of grain boundaries and formation of equiaxed nanosized grains.

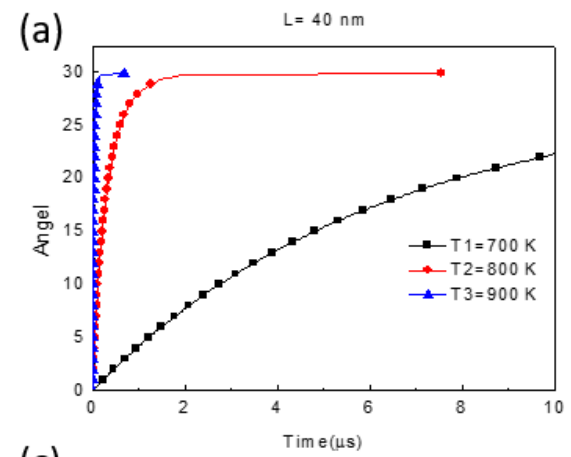

(c)

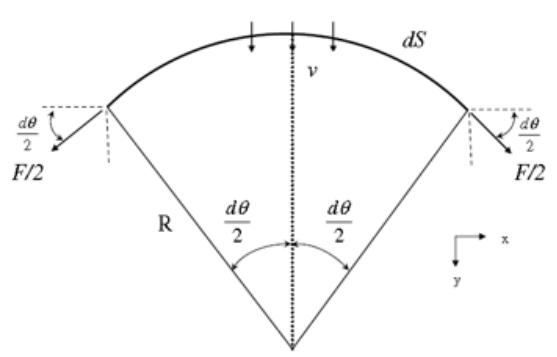

(b)

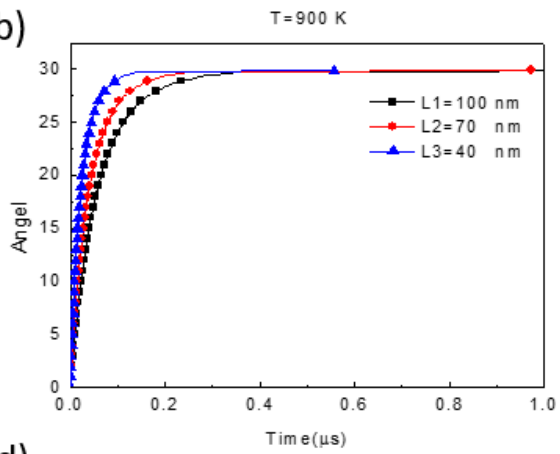

(d) 1

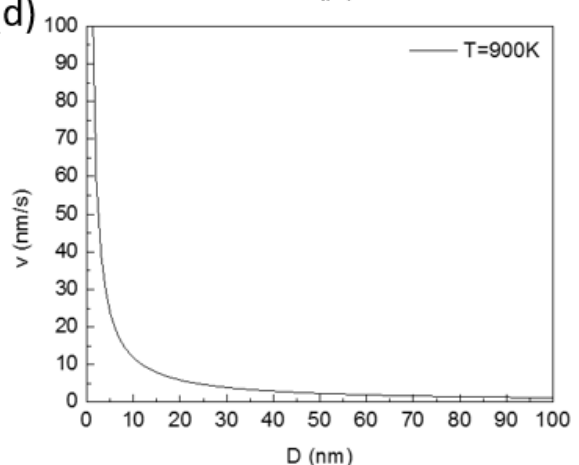

Figure 8. Angle of rotation of grain boundary during the deformation process as a function of time for (a) different grain sizes $(40,70$, and $100 \mathrm{~nm})$ at $\mathrm{T}=900 \mathrm{~K}$ and (b) different temperatures $(700,800$, and $900 \mathrm{~K})$ for grain size $\mathrm{L}=40 \mathrm{~nm}$. (c) Schematic showing force acting on the grain boundary with radius of curvature R. (d) Grain-boundary velocity as a function of radius R typical of shear band deformation process at $900 \mathrm{~K}$. 

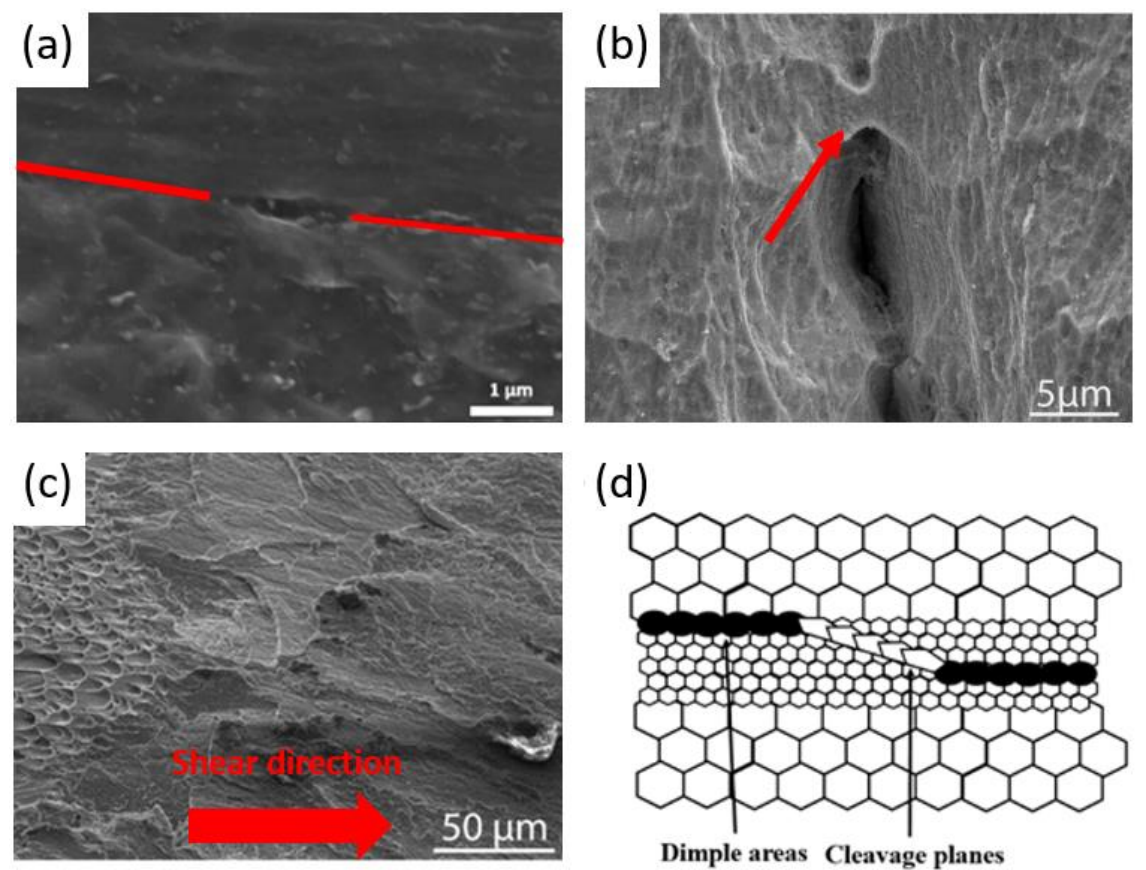

(d)

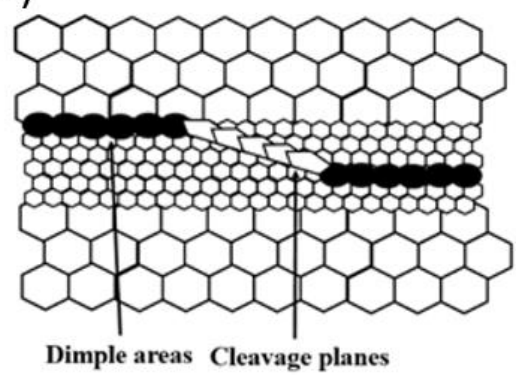

Figure 9. SEM images of the sample H-1 with max-displacement $1 \mathrm{~mm}$ (a) dimples along the shear band, (b) alignment between dimples and fracture surface, and of the sample $\mathrm{H}-2$ with max-displacement $1.15 \mathrm{~mm}(\mathrm{c})$ ductile areas and cleavage plane. (d) Schematic illustration of ASB failure of UFG titanium. 

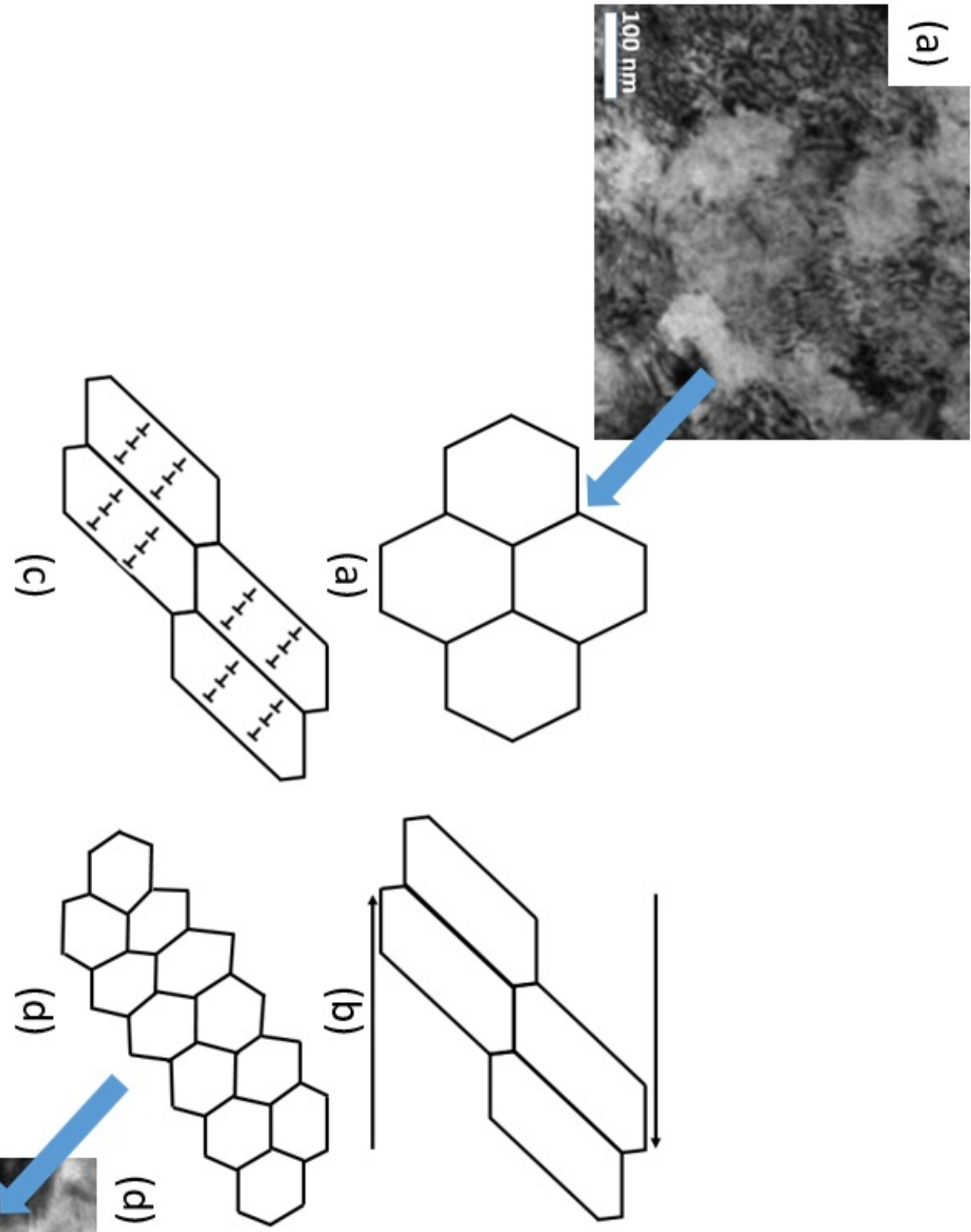